PROCEEDINGS OF THE

AMERICAN MATHEMATICAL SOCIETY

Volume 125, Number 11, November 1997, Pages 3289-3292

S 0002-9939(97)03990-7

\title{
LEMPERT MAPPINGS AND SYMPLECTIC FORMS
}

\author{
ZOLTAN BALOGH AND CHRISTOPH LEUENBERGER
}

(Communicated by Eric Bedford)

\begin{abstract}
We use Lempert's version of Riemann mapping to construct nonequivalent symplectic forms on an ellipsoid in $\mathbf{C}^{n}$.
\end{abstract}

Any bounded pseudoconvex domain $D \subseteq \mathbf{C}^{n}$ admits a strictly plurisubharmonic exhausting function $u$. If $u$ is smooth (or at least $C^{2}$ ) then $\omega=d d^{c} u=2 i \partial \bar{\partial} u$ is a symplectic form on $D$. The following theorem was proved in [2].

Theorem A (Eliashberg, Gromov). Let $u_{1}, u_{2}$ be two smooth strictly plurisubharmonic exhausting functions on a bounded strictly pseudoconvex domain $D$ :

$$
D=\left\{u_{1}<\infty\right\}=\left\{u_{2}<\infty\right\} .
$$

Then the forms $\omega_{1}=d d^{c} u_{1}$ and $\omega_{2}=d d^{c} u_{2}$ are symplectomorphic, i.e. there exists a diffeomorphism $\psi: D \rightarrow D$ such that $\psi^{*} \omega_{2}=\omega_{1}$.

As $u_{i}(z) \rightarrow \infty$ for $z \rightarrow \partial D$ it can easily be seen that the volume of $D$ with respect to the volume forms $\Omega_{i}=\omega_{i} \wedge \cdots \wedge \omega_{i}(i=1,2)$ is infinite. We will construct an example which shows that Theorem A no longer holds true for the finite volume case, i.e. when we give the domain not by exhausting but by defining functions.

Let $D$ be a bounded strictly pseudoconvex domain represented by a strictly plurisubharmonic smooth defining function $u_{1}$, i.e. $D=\left\{u_{1}(z)<0\right\} \subseteq \mathbf{C}^{n}$ with $\left.d u_{1}\right|_{\partial D} \neq 0$. $D$ is a symplectic manifold with the 2 -form $\omega_{1}=d d^{c} u_{1}$. Observe that the volume of $D$ with respect to the volume form $\Omega_{1}=\omega_{1} \wedge \cdots \wedge \omega_{1}$ is finite.

If $u_{2}$ is another defining function for $D$ that is equal to $u_{1}$ in a neighbourhood of the boundary $\partial D$ then $\int_{D} \Omega_{1}=\int_{D} \Omega_{2}$ as follows from Stokes' theorem. Moreover, using Moser's deformation argument (see e.g. [1], p.20) we can easily construct a symplectomorphism between $\left(D, \omega_{1}\right)$ and $\left(D, \omega_{2}\right)$. If $u_{1}$ and $u_{2}$ are not equal in a neighbourhood of $\partial D$ the volume condition alone is not sufficient to guarantee the existence of a symplectomorphism as will be shown in the following example.

The basis of our construction is Lempert's theorem stating that for any strictly convex domain $D \subseteq \mathbf{C}^{n}$ there is a homeomorphism of the unit ball $\rho: B \rightarrow D$ which has many similar properties to the usual Riemann mapping in $\mathbf{C}$. The property we need here is that the function $u: D \rightarrow R, u(z)=\frac{1}{4}\left(\left|\rho^{-1}(z)\right|^{2}-1\right)$ is a plurisubharmonic defining function of $D$ and moreover the relation $d d^{c} u=\rho_{*}^{-1} \omega_{1}$ holds where $\omega_{1}=\sum d y_{i} \wedge d x_{i}=\frac{1}{4} d d^{c}\left(|z|^{2}-1\right)$ is the standard symplectic form in $\mathbf{C}^{n}$ (see e.g. [5], Lemma 2.6).

Received by the editors May 20, 1996.

1991 Mathematics Subject Classification. Primary 32F05; Secondary 53C15.

Key words and phrases. Symplectic forms, Lempert mappings, pseudoconvex domains.

(C)1997 American Mathematical Society 
We are going to use furthermore the fundamental theorem of Gromov which is according to A. Weinstein the geometric expression of the uncertainty principle (see e.g. [4], p.358 for explanation). Gromov's theorem states that if there exists a symplectic embedding

$$
\left(B(r), \omega_{1}\right) \hookrightarrow\left(Z_{1}(R), \omega_{1}\right)
$$

of the ball in $\mathbf{R}^{2 n}$ of radius $r$ into the cylinder of radius $R$

$$
Z_{1}(R)=\left\{\left(x_{1}, y_{1}, \ldots, x_{n}, y_{n}\right) \in \mathbf{R}^{2 n}: x_{1}^{2}+y_{1}^{2}<R^{2}\right\}
$$

then necessarily $r \leq R$.

Let $A: \mathbf{C}^{2} \rightarrow \mathbf{C}^{2}, A\left(x_{1}, y_{1}, x_{2}, y_{2}\right)=\left(a x_{1}, a y_{1}, b_{1} x_{2}, b_{2} y_{2}\right)$ be a real linear map and $B=\{|z|<1\}$ be the unit ball in $\mathbf{C}^{2}$. Consider the real ellipsoid

$$
\begin{aligned}
E & =A^{-1}(B)=\left\{z \in \mathbf{C}^{2}:|A(z)|^{2}-1<0\right\} \\
& =\left\{x \in \mathbf{R}^{4}: a^{2} x_{1}^{2}+a^{2} y_{1}^{2}+b_{1}^{2} x_{2}^{2}+b_{2}^{2} y_{2}^{2}-1<0\right\} .
\end{aligned}
$$

We are going to construct two plurisubharmonic defining functions $u_{1}$ and $u_{2}$ for $E$ and consider the symplectic forms $\omega_{i}=d d^{c} u_{i}, i=1,2$. These will have the properties:

- $u_{i}$ are smooth and $\int_{E} \omega_{1} \wedge \omega_{1}=\int_{E} \omega_{2} \wedge \omega_{2}$;

- there exists no diffeomorphism $\psi: E \rightarrow E, \quad \psi^{*} \omega_{2}=\omega_{1}$.

First we choose the positive numbers $a, b_{1}, b_{2}$ carefully to fulfill the relations

1. $2 a^{2}=b_{1}^{2}+b_{2}^{2}$,

2. $a^{2} b_{1} b_{2}=1$,

3. $a^{2}>1$ and $b_{1} b_{2}<1$.

(For example, choose $a=2, b_{1}=\frac{1}{2 \sqrt{2}}(\sqrt{15}+\sqrt{17}), b_{2}=\frac{1}{4 b_{1}}$.)

The function $u_{1}(z)=\frac{1}{4 a^{2}}\left(|A(z)|^{2}-1\right)$ is a plurisubharmonic defining function for $E$ and by relation 1 we have $\omega_{1}=d d^{c} u_{1}=\sum_{i=1}^{2} d y_{i} \wedge d x_{i}$.

On the other hand we consider the Lempert mapping $\rho: B \rightarrow E, \rho(0)=0$, which exists as $E$ is strictly convex, and put $u_{2}(z)=\frac{1}{4}\left(\left|\rho^{-1}(z)\right|^{2}-1\right)$. Then $u_{2}$ is another plurisubharmonic defining function for $E$ and $\omega_{2}=\rho_{*}^{-1} \omega_{1}$ where $\omega_{1}$ is now the standard symplectic structure on the unit ball $B$. The forms $\omega_{1}$ and $\omega_{2}$ give the same volume for $E$. Indeed by relation 2 :

$$
\begin{gathered}
\operatorname{vol}_{\omega_{1}}(E)=\int_{E} \omega_{1} \wedge \omega_{1}=\int_{B}\left(A^{-1}\right)^{*}\left(\omega_{1} \wedge \omega_{1}\right)=\frac{1}{a^{2} b_{1} b_{2}} \int_{B} \omega_{1} \wedge \omega_{1}=\int_{B} \omega_{1} \wedge \omega_{1}, \\
\operatorname{vol}_{\omega_{2}}(E)=\int_{E} \omega_{2} \wedge \omega_{2}=\int_{\rho^{-1}(E)} \rho^{*}\left(\omega_{2} \wedge \omega_{2}\right)=\int_{B} \omega_{1} \wedge \omega_{1} .
\end{gathered}
$$

We claim that there is no symplectomorphism $\psi:\left(E, \omega_{2}\right) \rightarrow\left(E, \omega_{1}\right)$. For if such $\psi$ existed we could consider the map $\phi=\psi \circ \rho: B \rightarrow E$ which has the property $\phi^{*} \omega_{1}=\left(\rho^{*} \circ \psi^{*}\right) \omega_{1}=\rho^{*} \omega_{2}=\omega_{1}$.

On the other hand $E$ is contained in the cylinder

$$
Z_{1}\left(\frac{1}{a}\right)=\left\{x \in \mathbf{R}^{4}: x_{1}^{2}+y_{1}^{2}<\frac{1}{a^{2}}\right\} \subseteq \mathbf{C}^{2}
$$

and we have obtained an embedding

$$
\phi: B \hookrightarrow Z_{1}\left(\frac{1}{a}\right), \quad \phi^{*} \omega_{1}=\omega_{1} .
$$


But as $a>1$ by property 3 this is a contradiction to Gromov's theorem.

The deficiency of this construction is that the 2 -form $\omega_{2}=\rho_{*}^{-1} \omega_{1}$ might have discontinuous coefficients at $0 \in E$ as Lempert mappings $\rho$ are generally not smooth at the origin. But the situation can be rescued by pushing the singularity near the boundary with a suitable symplectic mapping.

To be more precise, denote by $B(r)$ the open ball of radius $r: B(r)=\{|z|<$ $r\} \subseteq \mathbf{C}^{n}$. As $\rho: B \rightarrow E$ is a homeomorphism and $\rho(0)=0$ we can choose a small neighbourhood $V$ of the origin in $E$ such that $\rho^{-1}(V) \subseteq B(\epsilon)$ for a given $\epsilon$. Applying standard convolution arguments we can smoothen $u_{2}$ to a $C^{\infty}$ plurisubharmonic function $\tilde{u}_{2}$ coinciding with $u_{2}$ outside of $V$.

By Stokes' theorem the volume of $E$ with respect to $\tilde{\omega}_{2}=d d^{c} \tilde{u}_{2}$ will still be the same. Now choose a compactly supported smooth Hamiltonian function $H$ on $B=B(1)$ with

$$
H=\left\{\begin{aligned}
x_{1} & \text { on } B(1-2 \epsilon) \\
0 & \text { on } B \backslash B(1-\epsilon) .
\end{aligned}\right.
$$

Let $h$ be the time- $(1-3 \epsilon)$-map of the Hamiltonian vectorfield $X_{H}$ defined by

$$
\iota_{X_{H}} \omega_{1}=d H
$$

As $X_{H}=\frac{\partial}{\partial y_{1}}$ on $B(1-2 \epsilon)$ the symplectomorphism $h$ maps the ball $B(\epsilon)$ around the origin into $B \backslash B(1-4 \epsilon)$.

Let us now assume the existence of a diffeomorphism $\psi: E \rightarrow E$ with $\psi^{*} \omega_{1}=\tilde{\omega}_{2}$. As before we put $\phi=\psi \circ \rho: B(1) \rightarrow E$ and now the mapping $\left.\phi \circ h^{-1}\right|_{B(1-4 \epsilon)}$ again yields a symplectic embedding

$$
\left(B(1-4 \epsilon), \omega_{1}\right) \hookrightarrow\left(Z_{1}\left(\frac{1}{a}\right), \omega_{1}\right) .
$$

Choosing $\epsilon$ small enough we arrive again at a contradiction to Gromov's theorem.

Thus we obtain

Theorem 1. There exist two smooth defining functions $u_{1}$ and $u_{2}$ of a strictly pseudoconvex domain $E$ such that their 2 -forms $\omega_{1}, \omega_{2}$ give rise to the same volume but they are not symplectically equivalent.

Let us finish this note with the following simple observation:

Proposition 1. Let $D \subseteq C^{n}$ be a complete circular domain with defining function $u$. If $d d^{c} u=\omega_{1}$ then $D$ is a ball.

Proof. Since $\omega_{1}=\sum d y_{i} \wedge d x_{i}=d d^{c} u_{0}$ for $u_{0}=\frac{1}{4} \sum\left|z_{i}\right|^{2}$ the function $h=u_{0}-u$ is pluriharmonic in $D$ and satisfies the following Dirichlet-type problem:

$$
\left\{\begin{array}{l}
d d^{c} h=0 \\
\left.h\right|_{\partial D}=\left.u_{0}\right|_{\partial D}
\end{array}\right.
$$

Let $L_{p}$ be a complex line through the origin and a point $p \in \partial D$. Then $L_{p} \cap D=$ $p \cdot \Delta=\{p \zeta: \zeta \in \Delta\}$ as $D$ is complete circular. But $\left.h\right|_{p \cdot \Delta}$ is harmonic and $\left.h\left|\partial(p \cdot \Delta)=\frac{1}{4}\right| p\right|^{2}$. Hence $h \equiv \frac{1}{4}|p|^{2}$, in particular $h(0)=u(0)=\frac{1}{4}|p|^{2}$. This proves that $|p|^{2}$ is independent of the choice of $p$. 


\section{ACKNOWLEDGEMENTS}

We would like to thank H.M. Reimann for valuable conversations concerning our example and the referee for his suggestions improving our presentation. Both authors are supported by the Swiss National Science Foundation.

\section{REFERENCES}

1. B. Aebischer, M. Borer, M. Kaelin, Chr. Leuenberger, H.M. Reimann, Symplectic Geometry, Progress in Mathematics, 124, Birkhaeuser 1994. MR 96a:58082

2. Y. Eliashberg, M. Gromov, Convex Symplectic Manifolds, Proceedings of Symposia in Pure Mathematics, 52 (1991), pp. 135-161.

3. L. Lempert, La métrique de Kobayashi et la représentation des domaines sur la boule, Bull. Soc. Math. France, 109 (1981), pp. 427-474. MR 84d:32036

4. D. McDuff, D. Salamon, Introduction to Symplectic Topology, Oxford Mathematical Monographs (1995). CMP 96:08

5. S. Semmes, A Generalization of Riemann Mappings and Geometric Structures on a Space of Domains in $C^{n}$, Memoirs of the AMS, 98, Nr. 472 (1992). MR 92k:32046

Mathematics Institute, University of Berne, Sidlerstrasse 5, 3012 Berne, SwitzerLAND

E-mail address: zoltan@math-stat.unibe.ch

E-mail address: leuenb@math-stat.unibe.ch 